\title{
Fate of a Bose-Einstein condensate with attractive interaction
}

\author{
Masahito Ueda ${ }^{1,2}$ and Kerson Huang ${ }^{1.3}$ \\ 1 Institute for Theoretical Physics, \\ University of California, Santa Barbara, CA 93106-4030 \\ 2 Department of Physical Electronics, \\ Hiroshima University, Higashi-Hiroshima 739-8257, Japan, and \\ Core Research for Evolutional Science and Technology (CREST), JST \\ ${ }^{3}$ Center for Theoretical Physics and Physics Department, \\ Massachusetts Institute of Technology, Cambridge, MA 02139 \\ MIT-CTP \# 2767 cond-mat/9807359
}

(November 6, 2017)

We calculate the decay amplitude of a harmonically trapped Bose-Einstein condensate with attractive interaction via the Feynman path integral. We find that when the number of particles is less than a critical number, the condensate decays relatively slowly through quantum tunneling. When the number exceeds the critical one, a "black hole" opens up at the center of the trap, in which density fluctuations become large due to a negative pressure, and collisional loss will drain the particles from the trap. As the black hole is fed by tunneling particles, we have a novel system in which quantum tunneling serves as a hydrodynamic source.

\section{Introduction}

Bose-Einstein condensation has been observed for the alkali atoms ${ }^{87} \mathrm{Rb}$ [1], ${ }^{23} \mathrm{Na}$ [2], and ${ }^{7} \mathrm{Li}$ [3], in magnetic trapping potentials at temperatures well below $10^{-6} \mathrm{~K}$. An important difference among these cases is that the scattering length between atoms is negative for ${ }^{7} \mathrm{Li}$, and positive for the others. This indicates a predominantly attractive interaction in the former case, and a repulsive one for the latter. Thus, we expect the ${ }^{7} \mathrm{Li}$ condensate to be metastable, and will decay with time. The purpose of this Letter is to elucidate the mechanism of the decay process.

To be sure, in all three cases the atoms will form metallic crystals in free space at low temperatures. This means that the short-range interactions are attractive in all cases, but the trapping potential tends to keep the atoms apart through zero-point motion, and in the case of positive scattering length, the atoms do not feel the attractive part of the potential.

We describe the many-boson system with a quantum field $\psi(\mathbf{r}, t)$, which is governed by the Hamiltonian [4]

$$
H=\int d^{3} r\left[\frac{\hbar^{2}}{2 m}|\nabla \psi|^{2}+\frac{1}{2} m \omega^{2} r^{2}|\psi|^{2}-\frac{u}{2}|\psi|^{4}\right]
$$

where $m$ is the atomic mass, $\omega$ is the frequency of the external harmonic potential, and $u=4 \pi \hbar^{2}|a| / m$ characterizes the interparticle interaction, with negative scattering length $a<0$. The transition amplitude from a given initial state $\psi_{0}(\mathbf{r})$ to some final state $\psi_{1}(\mathbf{r})$ in a time interval $t_{1}$ is given by the Feynman path integral

$$
\begin{aligned}
T & \equiv\left\langle\psi_{1}\left|\exp \left(-\frac{i}{\hbar} H t_{1}\right)\right| \psi_{0}\right\rangle \\
& =\int D \psi D \psi^{*} \exp \left(\frac{i}{\hbar} S[\psi]\right)
\end{aligned}
$$

with boundary conditions $\psi(\mathbf{r}, 0)=\psi_{0}(\mathbf{r})$ and $\psi\left(\mathbf{r}, t_{1}\right)=$ $\psi_{1}(\mathbf{r})$. The classical action $S[\psi]$ is

$$
\begin{aligned}
S[\psi]= & \int_{0}^{t_{1}} d t \int d^{3} r\left[i \hbar \psi^{*} \frac{\partial \psi}{\partial t}+\frac{\hbar^{2}}{2 m} \psi^{*} \nabla^{2} \psi\right. \\
& \left.-\frac{m \omega^{2} r^{2}}{2} \psi^{*} \psi+\frac{u}{2}\left(\psi^{*} \psi\right)^{2}\right]
\end{aligned}
$$

Physically, $\psi_{0}(\mathbf{r})$ and $\psi_{1}(\mathbf{r})$ correspond respectively to initial and final condensate wave functions, which are thermal averages of the quantum field. A Feynman path $\psi(\mathbf{r}, t)$ describes a possible history of the condensate wave function.

We calculate the transition amplitude in the semiclassical approximation, in the formal limit $\hbar \rightarrow 0$. The path integral can be evaluated in the saddle-point approximation. The saddle-point path, which corresponds to an extremum of the classical action, obeys the GrossPitaevskii (GP) equation with attractive interaction:

$$
i \hbar \frac{\partial \psi}{\partial t}=-\frac{\hbar^{2}}{2 m} \nabla^{2} \psi+\frac{m \omega^{2} r^{2}}{2} \psi-u \psi^{*} \psi^{2}
$$

We take as the initial condition $\psi_{0}(\mathbf{r})=C_{0} \exp \left(-r^{2} / 2 d^{2}\right)$, where $C_{0}=\sqrt{N / \pi^{3 / 2} d^{3}}$. The normalization is such that $\int d^{3} r \psi_{0}^{2}=N$, where $N$ is the total number of condensate bosons. The ground state wave function of the harmonic potential has the same form, but with $d=d_{0}$, where $d_{0}=$ $(\hbar / m \omega)^{1 / 2}$. For our initial state $d$ is a free parameter.

\section{The tunneling path}

The saddle-point path must be chosen to maximize, rather than minimize, the transition probability. Our Hamiltonian has no lower bound, and the wave function can grow without limit to attain an ever lower energy. If the initial wave function is small, however, it must first overcome an energy barrier presented by the zero-point kinetic term and the external potential. This it can do 
via quantum tunneling, which corresponds to a stationary path in imaginary time [5]7]. We tailor our initial discussion to the tunneling process, and put $t=-i \tau$. Self-consistency will tell us whether this is the right thing to do. The condensate wave function in imaginary time is denoted by $\phi(\mathbf{r}, \tau)=\psi(\mathbf{r},-i \tau)$, with complex conjugate $\phi^{*}(\mathbf{r}, \tau)=\psi^{*}(\mathbf{r}, i \tau)$. The final wave function $\phi_{1}$ is determined by the time evolution. In other words, we follow the path of least action. The transition probability is given by $|T|^{2} \approx A \exp \left(-2 S_{E} / \hbar\right)$, where $S_{E}$ is the Euclidean action of the saddle-point path:

$$
\begin{aligned}
S_{E}[\phi]= & \int_{0}^{T} d \tau \int d^{3} r\left[\hbar \phi^{*} \frac{\partial \phi}{\partial \tau}-\frac{\hbar^{2}}{2 m} \phi^{*} \nabla^{2} \phi\right. \\
& \left.+\frac{m \omega^{2} r^{2}}{2} \phi^{*} \phi-\frac{u}{2}\left(\phi^{*} \phi\right)^{2}\right]
\end{aligned}
$$

with $T=i t$. We shall not calculate the prefactor $A$ in this paper. Because our Hamiltonian has a translation invariance with respect to time, the transition probability should be proportional to $T$ (zero mode) as $T \rightarrow \infty$. Thus the transition rate is proportional to $\exp \left(-2 \hbar^{-1} S_{E}\right)$ to leading order.

To make all quantities dimensionless, we measure time in units of $\omega^{-1}$, distance in units of $d_{0}$. Thus, setting $\omega=d_{0}=1$, we have the imaginary-time GP equation

$$
-2 \frac{\partial \phi}{\partial \tau}=-\nabla^{2} \phi+r^{2} \phi-g|\phi|^{2} \phi
$$

where $g=4 \pi|a| / d_{0}$, with initial condition $\phi_{0}(\mathbf{r})=$ $C_{0} \exp \left(-\alpha r^{2} / 2\right)$, where $\alpha=\left(d_{0} / d\right)^{2}$.

Our problem is quite different from the relativistic case [6,7], because our equation is first-order in time and not second-order, and hence it describes diffusion rather than wave motion. Unlike the relativistic case, the motion here does not obey Newtonian mechanics in an inverted potential. We therefore do not have either the instanton [6] or the "bounce" [7].

We now make a modified "Thomas-Fermi approximation." Noting that initially we have $\nabla^{2} \phi_{0}=\left(\alpha^{2} r^{2}-\right.$ $3 \alpha) \phi_{0}$, we make the replacement $\nabla^{2} \phi \rightarrow\left(\alpha^{2} r^{2}-3 \alpha\right) \phi$. Assuming that $\phi$ is real, we can now rewrite the equation in terms of $\phi^{2}$ :

$$
\begin{aligned}
\frac{1}{2} \frac{\partial \phi^{2}}{\partial \tau} & =-V(r) \phi^{2}+g \phi^{4} \\
V(r) & =\frac{1}{2}\left[\left(1-\alpha^{2}\right) r^{2}+3 \alpha\right]
\end{aligned}
$$

The solution is given by

$$
\phi^{2}(r, \tau)=\frac{V(r)}{g\left[1+B(r) e^{2 \tau V(r)}\right]}
$$

where $B(r)$ is a monotonically increasing function of $r$ :

$$
B(r)=\frac{V(r)}{g \phi_{0}^{2}(r)}-1
$$

For large $r$ we have the asymptotic behavior $\phi(r, \tau) \approx$ $\phi_{0}(r) e^{-\tau V(r)}$.

\section{Critical number of condensate bosons}

If $B(0)>0$, then $\phi^{2}(r, \tau)$ will be positive and finite at all times, showing the self-consistency of the imaginarytime method. The condition that this be true is $3 \alpha / 2>$ $g C_{0}^{2}$, or $N<N_{c}$, with

$$
N_{c}=\frac{3 \sqrt{\pi} d}{8|a|} .
$$

The total decay rate is $\exp \left(-2 S_{E} / \hbar\right)$, where

$$
\frac{S_{E}}{\hbar}=\frac{\pi}{g} \int_{0}^{\infty} d r r^{2} V(r)\left[\ln \frac{1}{1-f(r)}-f(r)\right]
$$

where

$$
f(r)=\frac{g \phi_{0}^{2}(r)}{V(r)}=\frac{N}{N_{c}} \frac{\exp \left(-\alpha r^{2}\right)}{1+r^{2}\left(1-\alpha^{2}\right) /(3 \alpha)}
$$

The critical number of condensate bosons $N_{c}$ has been calculated with various methods 8 10, and all results are of the same order of magnitude, and not in disagreement with experimental indications [11]. Previous estimates of the tunneling probability, however, give varied physical pictures and mathematical results [12 15]. Our result is closest to that of Ref. 15.

At each point $r$ in the trap, particles tunnel through an energy barrier that depends on $r$. Consequently, they "sweat out" at different rates at different points in space. Once over the energy barrier, the saddle-point path veers onto the real time axis, and the particles evolve according to the PG equation in real time. We shall comment on their actual fate later.

\section{The black hole}

When $N>N_{c}$ the initial wave function near the origin is large enough to go over the energy barrier. This part of the wave function will evolve according to the PG equation in real time, and we expect that density fluctuations will become large, with concomitant collisional loss. Thus, a "black hole" opens up near the center of the potential, where particles will be drained at a rapid rate. In the imaginary-time formalism, the existence of the black hole is indicated by the fact that $B(r)$ is negative at the origin, and increases to zero at $r=b$. The black hole radius $b$ is determined by the transcendental equation

$$
\phi_{0}^{2}(b)=g^{-1} V(b)
$$

There is no root unless $N>N_{c}$. Near the critical value we have $b^{2} \approx c_{0}\left(N-N_{c}\right) / N_{c}$, where $c_{0}=3 \alpha /\left(1+2 \alpha^{2}\right)$, and for large $N, b^{2}$ increases like $\ln N$.

According to our solution, the condensate wave function is independent of $\tau$ at the black hole radius, decreases with $\tau$ at any point outside the radius, and increases with $\tau$ inside. Inside the black hole, the wave 
function increases rapidly with decreasing distance, and diverges at $r=r_{1}(\tau)$, determined by the condition $1+B \exp (2 \tau V)=0$. As $\tau$ increases, $r_{1} \rightarrow b$ at an exponential speed, so that $\phi^{2}$ quickly becomes uniformly divergent just inside the rim of the black hole. Further into the black hole, $\phi^{2}$ becomes negative. The last fact indicates that the imaginary-time formalism is inconsistent inside the black hole, and we should go to a real-time description.

\section{Negative pressure and instability}

Reverting to real time inside the black hole, we write $\psi(\mathbf{r}, t)=\sqrt{n} e^{i \theta}$, and obtain a pair of hydrodynamic-like equations [16]. One of these is the continuity equation $\partial n / \partial t+\nabla \cdot(n \nabla \theta)=0$, and the other reads $\partial \theta / \partial t+\Omega=0$, where

$$
\Omega=\frac{1}{2}(\nabla \theta)^{2}+\frac{1}{2}\left(r^{2}-\frac{1}{\sqrt{n}} \nabla^{2} \sqrt{n}\right)-g n
$$

Using the modified Thomas-Fermi approximation, we replace the second term on the right side by $V(r)$. The steady-state solution $\partial \theta / \partial t=0$ gives $\Omega=0$, or $\frac{1}{2} v_{s}^{2}+V-g n=0$, where $\mathbf{v}_{s}=\nabla \theta$ is the superfluid velocity. This is like Bernoulli's law in hydrodynamics, but with negative pressure $P=-g n$. Solving for the superfluid velocity we obtain

$$
v_{s}=\sqrt{2[\operatorname{gn}(r)-V(r)]}
$$

The region in which this is real is precisely the black hole. Outside this region $v_{s}$ becomes pure imaginary, and we are back in the tunneling regime.

Since the pressure is negative, however, the system must be highly unstable with respect to density fluctuations, which were ignored in our modified Thomas-Fermi approximation. The instability must lead to nonuniform collapse into pockets of extremely high density, with superfluid turbulence. We must also take into account the fact that inelastic collisions, such as three-body molecular recombinations, become increasingly likely with increasing density, and these collisions will expel particles from the trap [12,17]. This region is therefore a cauldron of density fluctuations and collisional loss, and truly a black hole.

\section{Outlook}

The trapped Bose-Einstein condensate with attractive interaction offers many exciting possibilities for theoretical and experimental study.

(1) The opening and closing of a black-hole region, whose size depends on the number of particles, furnishes a mechanism for the phenomenon of condensate oscillation suggested recently 18,19]. A quantitative study will necessitate a dynamical treatment.

(2) In a kinetic theory, we expect that the particles "sweating" out throughout the trap, due to tunneling, will create a steady flow into the black hole, and drain out eventually due to collisions. Here, for the first time, we have a system in which macroscopic tunneling serves as hydrodynamic source. The kinetic theory in this case will have to bridge quantum tunneling and fluid mechanics.

(3) By plugging the black hole with a laser beam of variable size, one can create a "faucet" that controls the draining of the condensate. The remaining condensate, which decays slowly via tunneling, should support a larger maximum number.

We thank Randy Hulet for sharing with us some of his data before publication, the organizers of the Workshop on Bose-Einstein condensation at the Institute for Theoretical Physics at Santa Barbara, 1998, for making this work possible, and the participants of the Workshop for discussions. One of us (KH) was supported in part by DOE cooperative agreement DE-FC02-94ER40818.

[1] M.H. Anderson, J.R. Ensher, M.R. Mathews, C.E. Wieman, and E.A. Cornell, Science 269, 198 (1995).

[2] K.B. Davis, M.-O. Mewes, M.R. Andrews, N.J. van Druten, D.S. Durfee, D.M. Kurn, and W. Ketterle, Phys. Rev. Lett. 75, 3969 (1995).

[3] C.C. Bradley, C.A. Sackett, J.J. Tollet, and R.G. Hulet, Phys. Rev. Lett. 75, 1687 (1995); C.C. Bradley, C.A. Sackett, and R.G. Hulet, Phys. Rev. Lett. 78, 985 (1997).

[4] See, for example, K. Huang, Statistical Mechanics, 2nd ed (Wiley, New York, 1987).

[5] J.S. Langer, Ann. Phys. (NY), 41, 108 (1967).

[6] G. 'tHooft, Phys. Rev. Lett. 37, 8 (1976).

[7] S. Coleman, Phys. Rev. D15, 2929 (1977).

[8] P.A. Ruprecht, M. J. Holland, K. Burnett, and M. Edwards, Phys. Rev. A 51, 4704 (1995).

[9] G. Baym and C.J. Pethick, Phys. Rev. Lett. 76, 6 (1996).

[10] F. Dalfovo and S. Stringari, Phys. Rev. A53, 2477 (1966).

[11] C.C. Bradley, C.A. Sackett, and R.G. Hulet, Phys. Rev. Lett. 78, 985 (1997).

[12] Yu. Kagan, G. Shlyapnikov, and J. Walraven, Phys. Rev. Lett. 76, 2670 (1966).

[13] E. Shuryak, Phys. Rev. A54, 3151 (1996).

[14] H.T.C. Stoof, J. Stat. Phys. 87, 1353 (1997).

[15] M. Ueda and A.J. Leggett, Phys. Rev. Lett. 80, 1576 (1998).

[16] For a review see F. Dalfovo, S. Giorgini, L.P. Pitaevskii, and S. Stringari, Rev. Mod. Phys. (to be published).

[17] R.J. Dodd, M. Edwards, C. Williams, C.W. Clark, M. Holland, P. Ruprecht, and K. Burnett, Phys. Rev. A 54, 661 (1996).

[18] C.A. Sackett, H.T.C. Stoof, and R.G. Hulet, Phys. Rev. Lett. 80, 2031 (1998).

[19] Yu. Kagan, A.E. Muryshev, and G.V. Shlyapnikov, condmat/9801168. 